\title{
S-Propargyl-Cysteine, a Novel Water-Soluble Modulator of Endogenous Hydrogen Sulfide, Promotes Angiogenesis Through Activation of Signal Transducer and Activator of Transcription 3
}

\author{
Juntao Kan, ${ }^{1, *}$ Wei Guo, ${ }^{2, *}$ Chengrong Huang, ${ }^{2}$ Guangzhi Bao, ${ }^{2}$ Yichun Zhu, ${ }^{3}$ and Yi Zhun Zhu ${ }^{1,4}$
}

\begin{abstract}
Aims: Conventional revascularization strategies or drug therapies for ischemic heart disease (IHD) are designed for reperfusion of coronary arteries to salvage cardiomyocytes, but occasionally, myocardial reperfusion injury can occur because of microcirculatory dysfunction. Therefore, a more microcirculation-friendly strategy should be explored to overcome and compensate for the shortcomings of conventional strategies. In this work, we investigated the proangiogenic effect of S-Propargyl-Cysteine (SPRC), a novel water-soluble modulator of endogenous hydrogen sulfide, and elucidated the possible mechanisms involved to provide an experimental basis for angiogenesis-mediated drug therapy for IHD. Results: SPRC promoted cell proliferation, adhesion, migration, and tube formation of primary human umbilical vein endothelial cells (HUVEC) and increased angiogenesis in the rat aortic ring and Matrigel plug models. In a mouse model of hindlimb ischemia and a rat model of myocardial ischemia, SPRC also promoted angiogenesis after ligation of the left femoral artery or coronary artery to ameliorate ischemic conditions. In primary HUVEC, STAT3 phosphorylation was significantly induced after SPRC treatment. The critical roles of STAT3 in mediating the proangiogenic effect of SPRC were confirmed by RNA interference. Co-crystallization excluded the possible direct interaction between SPRC and STAT3, whereas co-immunoprecipitation revealed an enhanced interaction between VEGFR2 and STAT3 after SPRC treatment. Meanwhile, immunofluorescence and chromatin immunoprecipitation showed that SPRC induced the nuclear translocation of STAT3, followed by transcriptional activation of downstream promoters, particularly the Vegf promoter. Innovation and Conclusion: We present a novel STAT3-mediated mechanism in SPRCinduced angiogenesis and demonstrate the therapeutic potential of SPRC in ischemic disease through angiogenesis promotion. Antioxid. Redox Signal. 20, 2303-2316.
\end{abstract}

\section{Introduction}

A S REPORTED BY the World Health Organization (WHO), ischemic heart disease (IHD) is the leading cause of death in both developed and developing countries (21). Conventional revascularization strategies, such as primary percutaneous coronary intervention (PCI), focus on prompting reperfusion of coronary arteries to salvage cardiomyocytes,

\section{Innovation}

We present a novel STAT3-mediated angiogenic mechanism that is induced by S-propargyl-cysteine (SPRC), a novel modulator of endogenous hydrogen sulfide, and demonstrate the therapeutic potential of SPRC in ischemic disease through the promotion of angiogenesis, which sheds new light on hydrogen sulfide-mediated drug therapy.

\footnotetext{
${ }^{1}$ Department of Pharmacology, School of Pharmacy and Institutes of Biomedical Sciences, Fudan University, Shanghai, China.

${ }^{2}$ Department of Pharmacology, School of Pharmacy, Fudan University, Shanghai, China.

${ }^{3}$ Department of Physiology and Pathophysiology, Shanghai Medical College, Fudan University, Shanghai, China.

${ }^{4}$ Department of Pharmacology, Loo Yong Lin School of Medicine, National University of Singapore, Singapore, Singapore.

*Both authors contributed equally to this work.
} 
but they have been suspected to result in microcirculatory dysfunction (29). Therefore, a more positive angiogenesismediated recovery of the microvasculature that is stimulated by pharmacological or genetic intervention may have a greater potential in IHD therapy $(29,36)$.

Angiogenesis is a physiological or pathological process that is characterized by the sprouting of new blood vessels from existing vessels (6). Vascular endothelial growth factor (VEGF) is the crucial regulator of angiogenesis that primarily binds to two related receptor tyrosine kinases (RTKs), known as VEGFR1 (Flt-1) and VEGFR2 (KDR or Flk-1) $(8,20)$. Compared with VEGFR1, VEGFR2 plays a more vital role in angiogenesis and is a major mediator of VEGF's biological effects.

Signal transducer and activator of transcription (STAT) is an important family of nuclear factors (NFs), and it was originally found to be activated by cytokine receptors, particularly interferon (IFN) and interleukin-6 (IL-6) receptors via Janus kinase (JAK) (15). STAT can also be activated by RTKs, such as epidermal growth factor receptor and VEGFR (38). STAT3 is an extensively investigated NF from the STAT family, and it exerts crucial effects on angiogenesis in both cancer formation (19) and heart pathogenesis (12). STAT3 and VEGF/VEGFR2 have been investigated in endothelial cells and cardiomyocytes to disclose the mutual promoting effects between them $(2,22,34)$. As a potential molecular target of angiogenesis-mediated therapy, STAT3 has received more attention in the cardiovascular system $(12,22)$.

First discovered in the cardiovascular (37) and nervous systems (1), the physiological effects of hydrogen sulfide are attracting more research interest $(23,25)$. In the cardiovascular system, hydrogen sulfide plays an important positive role in myocardial ischemia attenuation $(5,39)$. Furthermore, the proangiogenic effect of hydrogen sulfide was successively discovered by two different groups $(4,24,30)$. Although many protective effects have been clarified, hydrogen sulfide cannot be used for clinical therapy because of its gaseous nature. S-propargyl-cysteine (SPRC, also named as ZYZ-802), which was designed and synthesized by our group, is a novel watersoluble modulator of endogenous hydrogen sulfide $(31,32)$. SPRC promotes the activity of cystathionine- $\gamma$-lyase $(\mathrm{CTH}$, also named CSE, producing enzymes of endogenous hydrogen sulfide) and then elevates hydrogen sulfide levels in plasma or tissue (31). In our previous study, we reported the protective effects of SPRC on rats with IHD via an antioxidation mechanism $(14,32)$. Here, we proposed and tested SPRC-induced angiogenesis both in vitro and in vivo. In addition, we investigated the relative mechanisms of STAT3, including its interaction with VEGFR2 and transcriptional activation, in human umbilical vein endothelial cells (HUVEC).

\section{Results}

\section{SPRC promoted primary HUVEC proliferation, adhesion, migration, and tube formation}

We treated primary HUVEC with different SPRC concentrations to assess a possible proangiogenic effect in vitro. SPRC administration (from 10 to $100 \mu \mathrm{M}$ ) significantly increased primary HUVEC viability (Fig. 1A). In addition, SPRC (100 $\mu M)$ promoted cell proliferation, but this effect could be abolished by propargylglycine (PAG), a CTH inhibitor (Fig. 1B and Supplementary Fig. S1; Supplementary Data are available online at www.liebertpub.com/ars). We next investigated the effects of
SPRC on primary HUVEC adhesion, migration, and tube formation and demonstrated that SPRC $(100 \mu M)$ could be responsible for promoting these functions (Fig. 1C). In adhesion assay, an incubation time of only 10 min was sufficient for SPRCinduced adhesion of primary HUVEC, suggesting that SPRC may act through a swift response mechanism. Wound healing and transwell assays were performed to investigate migration, and the results of both demonstrated a significant promoting effect of SPRC on primary HUVEC migration. In a 3D Matrigel assay, primary HUVEC were cultured to form tube structures, and the tube length increased after SPRC treatment (Fig. 2).

\section{SPRC promoted angiogenesis in vivo under both normal and ischemic conditions}

Based on the outstanding proangiogenic effects of SPRC in vitro, we continued to investigate the possible effects ex vivo and in vivo. We prepared rat aorta rings to be embedded in Matrigel and determined that branch-like microvessels outgrew considerably more after SPRC treatment for 6 days (Fig. 3A). However, PAG abolished the positive effect of SPRC. Likewise, angiogenesis was also promoted in Matrigel plugs implanted in mice. After SPRC administration $(50 \mathrm{mg} / \mathrm{kg} /$ day) for 7 days, more endothelial cells invaded deeper into the Matrigel plug, with more mature capillary vessels observed after staining by CD31 antibody (6.00-fold, Fig. 3B-D). In addition, the hemoglobin concentration was significantly increased in Matrigel plugs from animals treated with SPRC (4.19-fold, Fig. 3E). Three in vivo angiogenesis models were constructed under nonischemic condition to obtain similar results (Supplementary Fig. S2).

To investigate the possible therapeutic potential of SPRC in ischemic disease, we tested a mouse model of hindlimb ischemia and a rat model of myocardial ischemia. After femoral artery ligation, blood flow in the ischemic hindlimb was significantly reduced (Fig. 4A). A serial analysis of hindlimb perfusion by laser Doppler imaging revealed significantly more flow recovery in SPRC-treated mice $(50 \mathrm{mg} / \mathrm{kg} /$ day $)$ compared with vehicle-treated mice $(0.85 \pm 0.05$ vs. $0.71 \pm 0.10$ on day $3,0.94 \pm 0.10$ vs. $0.75 \pm 0.08$ on day 7 ). Subsequently, we performed microangiography using barium sulfate to develop collateral vessels. Ligation led to collateral remodeling in the ischemic hindlimb, which was consistent with a previous report (7). After SPRC treatment, the angiographically visible collateral vessels were significantly increased, some with typical "corkscrew" appearance (Fig. 4B). Using a specific endothelial cell marker (CD31), it was found that SPRC also caused a significant increase in gastrocnemius muscle capillary density (1.75-fold, Fig. 4C), suggesting that the increase in microvessels may contribute to hindlimb blood supply for ischemic condition attenuation. Likewise, in the rat model of coronary artery ligation, angiographic data showed increased vascular density (1.68-fold) of ischemic heart in SPRC-treated rats compared with that in vehicle-treated rats (Fig. 4D). Taken together, these in vivo results suggest that SPRC have therapeutic potential in ischemic disease through the promotion of angiogenesis or arteriogenesis to ameliorate ischemia-induced pathology.

\section{The proangiogenic effect of SPRC was mediated by STAT3}

We first determined that SPRC induced STAT3 phosphorylation in primary HUVEC in a time- and dose-dependent 
A

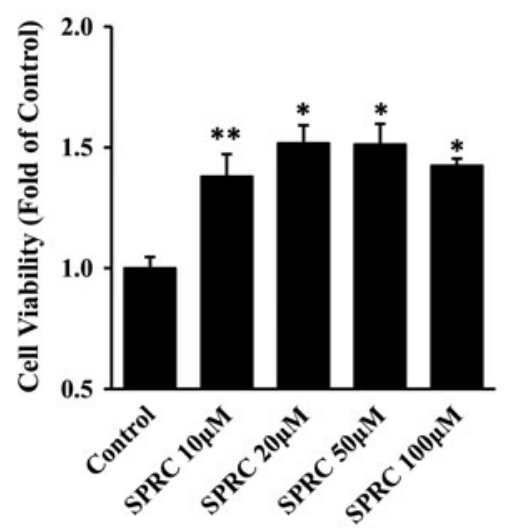

B

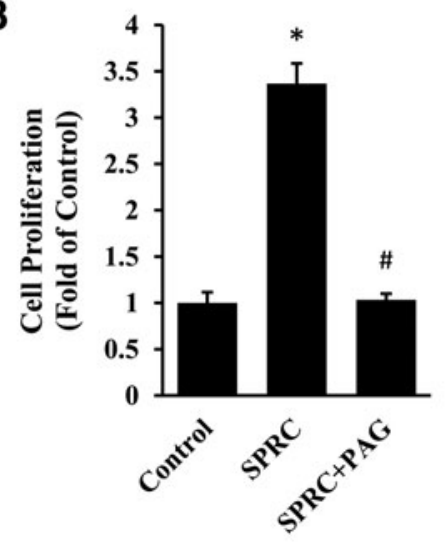

C
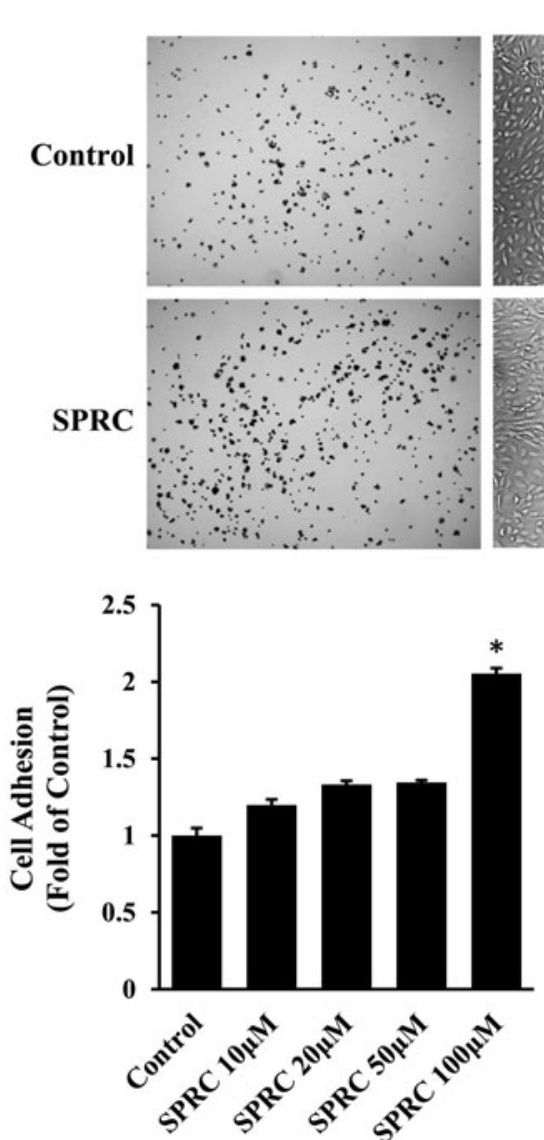

Migration
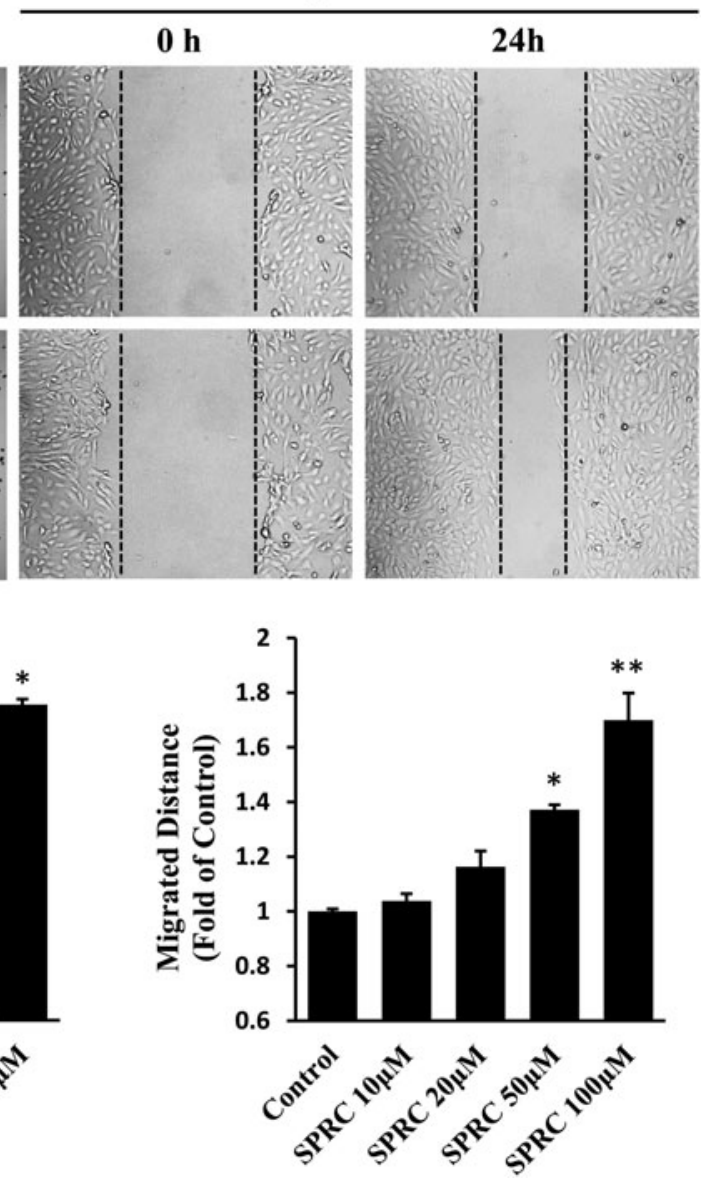

FIG. 1. S-propargyl-cysteine (SPRC) promoted cell viability, proliferation, adhesion, and migration of primary human umbilical vein endothelial cells (HUVEC). (A) Cell viability assay. Primary HUVEC were treated with different concentrations $(10,20,50$, and $100 \mu \mathrm{M})$ of SPRC for $24 \mathrm{~h}$ and then analyzed by MTT assay. (B) Cell proliferation assay. Primary HUVEC were treated with different drugs for $24 \mathrm{~h}$ and then stained by EdU and DAPI. The red nuclei indicating proliferated cells were counted and statistically analyzed. Propargylglycine (PAG) (1 mM) is an inhibitor of cystathionine- $\gamma$-lyase (CTH). (C) Cell adhesion and migration assay. For adhesion assay, primary HUVEC were seeded in collagen I-precoated wells and treated with different SPRC concentrations for $10 \mathrm{~min}$. For wound-healing assay, cell monolayers were scraped and treated with different SPRC concentrations for $24 \mathrm{~h} .{ }^{*} p<0.01$ versus control group, ${ }^{* *} p<0.05$ versus control group, and ${ }^{\#} p<0.01$ versus SPRC-treated group. All experiments were repeated at least thrice.

manner (Fig. 5A, B). In addition, these effects could be abolished by WP1066, an inhibitor of STAT3 (Fig. 5C). Next, we transfected STAT3 siRNA into primary HUVEC to silence STAT3 expression (Fig. 5D) and discovered the crucial role of STAT3 in SPRC-induced angiogenesis in vitro. After transfection with STAT3 siRNA, primary HUVEC adhesion, migration, and tube formation were significantly inhibited (Fig. 2). In the control siRNA group, SPRC promoted primary HUVEC adhesion, migration, and tube formation. However, in the STAT3 siRNA group, the deficit of STAT3 expression abolished the promoting effects of SPRC on adhesion and tube formation.

\section{The interaction between VEGFR2 and STAT3 was enhanced after SPRC treatment}

To investigate the mechanism of STAT3 activation, we first performed co-crystallization between STAT3 and SPRC to test whether STAT3 could be directly activated by SPRC. After soaking in SPRC solution, the electron densities around STAT3 cysteine residues were still clear, and no significant extra-electron densities were observed (Supplementary Fig. S3), suggesting that there may be no direct interaction between SPRC and STAT3.

Transformed cells of human umbilical vein endothelium (HUV-EC-C) were used to replace primary HUVEC for investigating VEGFR2-related protein interactions. Reciprocal co-immunoprecipitation revealed that the interaction between VEGFR2 and Grb2, a growth factor receptor-bound protein, was enhanced after SPRC treatment (Supplementary Fig. S4), suggesting the possible activation of signaling pathways downstream of VEGFR2. Next, we studied the interaction between VEGFR2/Grb2 and JAK-STAT3 pathway. After precipitation with VEGFR2 or Grb2 antibody, complexes were analyzed by Western blot. We found that both JAK and STAT3 acted more positively with VEGFR2 after SPRC treatment (Fig. 6A), whereas STAT3 acted negatively with Grb2 after SPRC treatment. The results suggested that after 

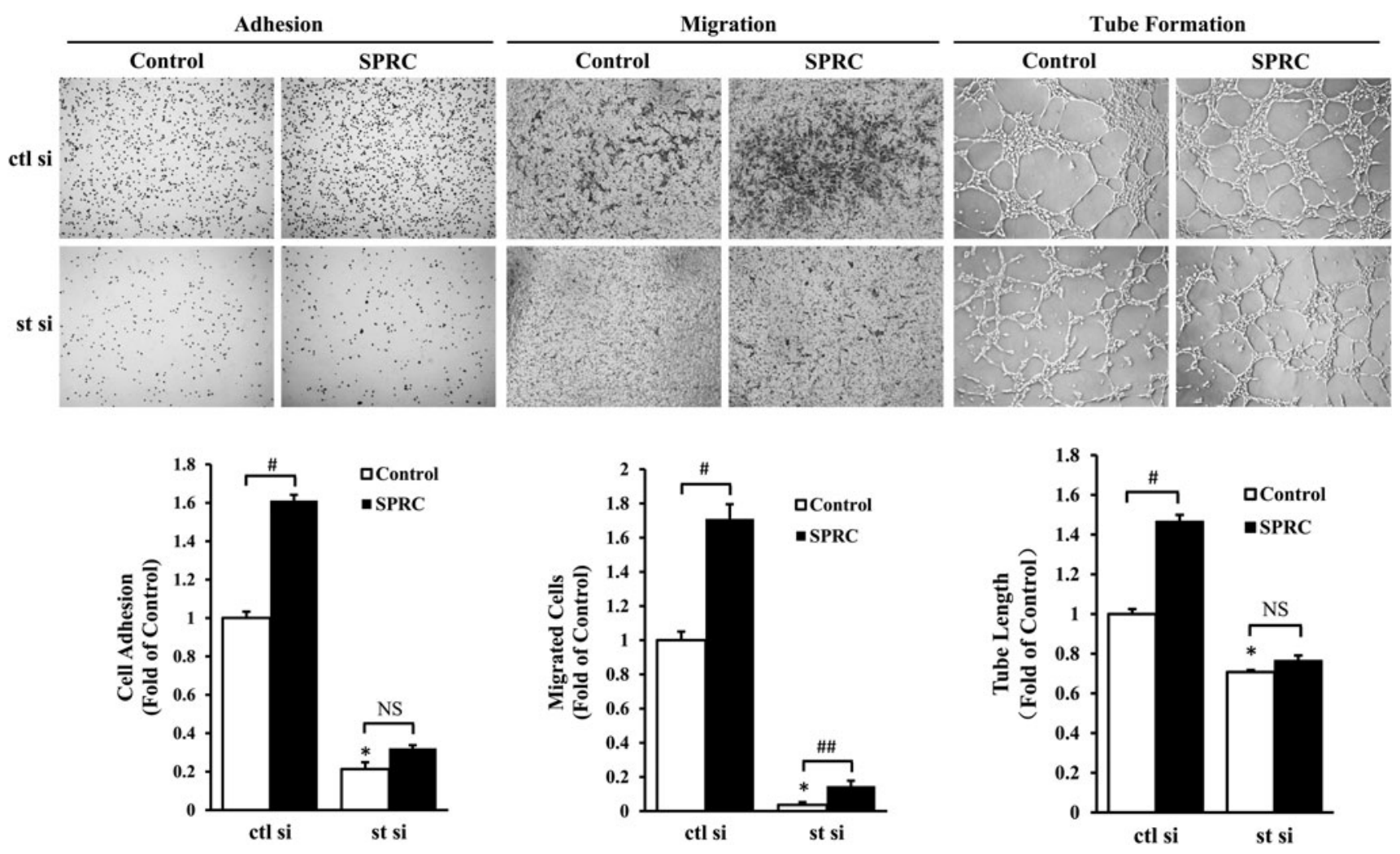

FIG. 2. The proangiogenic effect of SPRC was mediated by STAT3. For adhesion assay, primary HUVEC were seeded in collagen I-precoated wells and treated with SPRC $(100 \mu M)$ for $10 \mathrm{~min}$. For transwell migration assay, primary HUVEC were seeded in the upper compartment of the transwell, while SPRC $(100 \mu \mathrm{M})$ was added to the lower compartment. Cells were treated for $8 \mathrm{~h}$. For tube formation assay, primary HUVEC were seeded on the gelled Matrigel and treated with SPRC $(100 \mu \mathrm{M})$ for $6 \mathrm{~h}$. STAT3 silencing was performed by siRNA transfection using an RNAiMAX kit for $48 \mathrm{~h}$ before SPRC treatment. Control siRNA containing a scrambled sequence was used as a negative control. ctl si, control siRNA; st si, STAT3 siRNA. ${ }^{*} p<0.01$ versus control group, ${ }^{\#} p<0.01$ between groups as indicated, ${ }^{\# \#} p<0.05$ between groups as indicated. NS, nonsignificant $(p>0.05)$. All experiments were repeated at least thrice.

SPRC treatment, STAT3 was prompted to assemble at VEGFR2 binding sites to transmit a possible VEGFR2generated signal. Double fluorescent labeling showed that STAT3 was co-localized with VEGFR2 (Fig. 6B). Furthermore, treatment with SU5416, a selective tyrosine-kinase inhibitor of VEGFR2, inhibited SPRC-induced STAT3 phosphorylation (Fig. 6C), suggesting the possible signal transduction from VEGFR2 to STAT3. Likewise, VEGFR2 siRNA abolished the SPRC-induced STAT3 phosphorylation (Supplementary Fig. S5).

\section{SPRC induced the nuclear translocation of STAT3, followed by transcriptional activation}

To investigate how STAT3 activated downstream molecules, cytoplasmic and nuclear proteins from HUV-EC-C were isolated, and we detected an increase in both nuclear STAT3 expression and phosphorylation, with little change in cytoplasmic levels (Fig. 7A). Corresponding with the Western blot results, a significant STAT3 translocation from the cytosol to the nucleus was observed in primary HUVEC by confocal microscopy after SPRC treatment (Fig. 7B). To determine STAT3 activity in the nucleus, we performed an electrophoretic mobility shift assay (EMSA) using HUV-EC-C nuclear extracts. A much brighter shift band appeared in the SPRC- treated group compared with the control group; however, such promoting effects could be abolished by WP1066 (Fig. 7C). To investigate the STAT3-activated gene promoters, we performed chromatin immunoprecipitation (ChIP) with HUV-EC-C nuclear extracts. The promoters of Vegf, Akt, Erk, and $C$ th were significantly activated after SPRC treatment (Fig. 7D), and their activation, particularly of the Vegf promoter, may be directly responsible for SPRC-induced angiogenesis in endothelial cells. We observed a time-dependent increase in protein synthesis of VEGF, Akt, Erk, and CTH several hours after SPRC treatment (Supplementary Fig. S6). Furthermore, in two separate in vivo experiments, VEGF and $\mathrm{CTH}$ were confirmed to be more abundant in SPRC-treated animals (Supplementary Fig. S7).

\section{Discussion}

Conventionally, patients with IHD are always treated using pharmacological therapies, such as antiplatelet agents (aspirin), antilipidemic agents (statins) or vasodilators, or revascularization strategies, including PCI or coronary artery bypass graft surgery, for reperfusion of coronary arteries to salvage cardiomyocytes. However, a phenomenon termed myocardial reperfusion injury occasionally appears as a result of microcirculatory dysfunction that is primarily induced by oxidative 

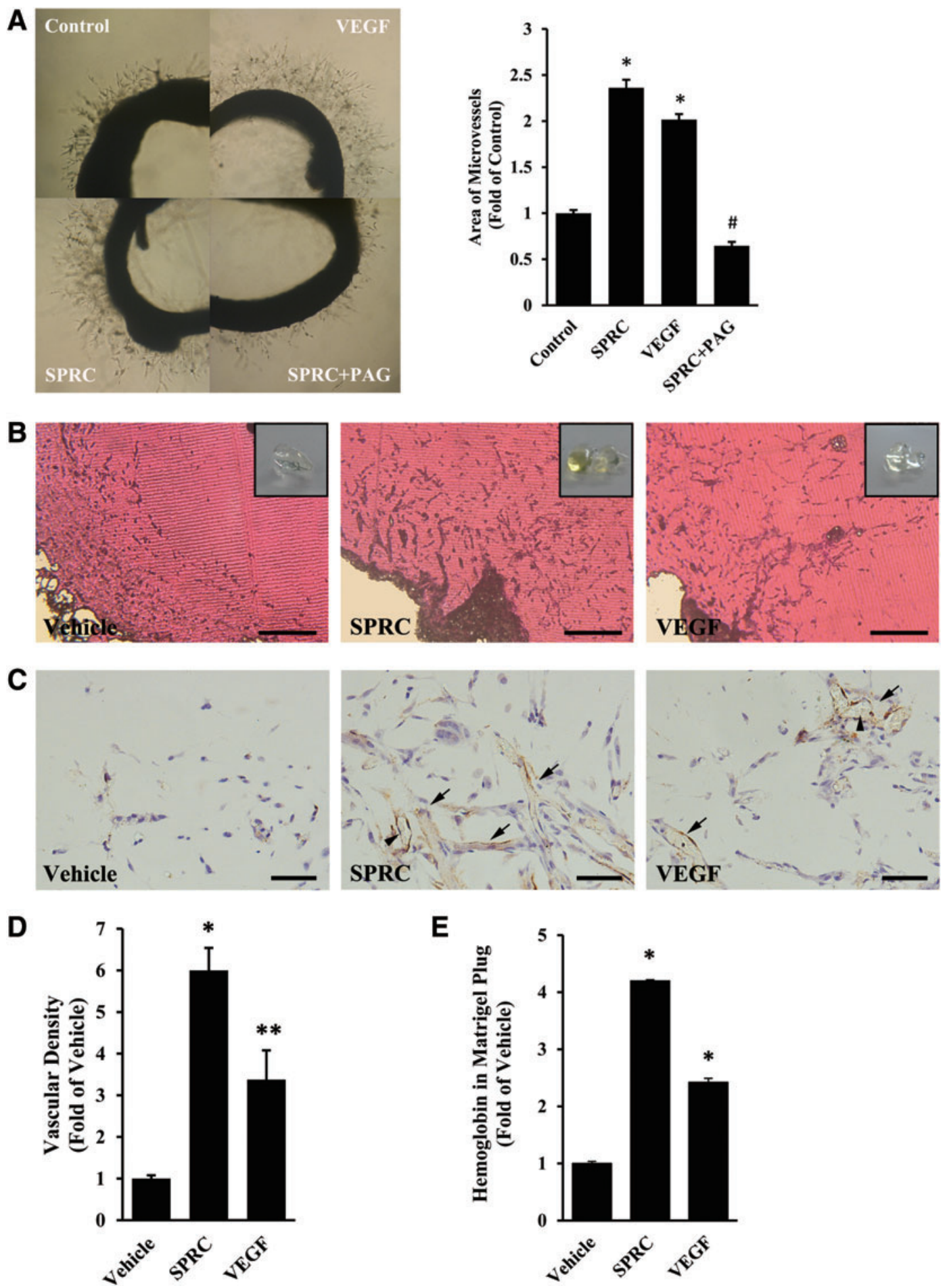

FIG. 3. SPRC promoted angiogenesis ex vivo and in vivo under normal conditions. (A) Aortic ring assay. Rat aortic rings were embedded in Matrigel and then treated with vehicle, SPRC (100 $\mu \mathrm{M})$, VEGF (2 ng/ml), and SPRC plus PAG $(100 \mu M+1 \mathrm{mM})$ for 6 days. ${ }^{*} p<0.01$ versus control group, ${ }^{\#} p<0.01$ versus SPRC-treated group. (B-E) Matrigel plug assay. After Matrigel implantation, mice in the SPRC-treated group were injected intraperitoneally with SPRC (50 mg/kg/day) for 7 days. Matrigel plugs were used for morphological analyses or determination of hemoglobin concentration. VEGF (100 ng/ml) was premixed with Matrigel before implantation. $n=10$ for each group. (B) Hematoxylin-eosin staining. A fresh Matrigel plug is shown in the inset. Scale bar, $400 \mu \mathrm{m}$. (C) Immunohistochemistry. CD31-positive capillary vessels are indicated by solid arrows. Note the erythrocytes in the vessels (arrowhead). Scale bar, $50 \mu \mathrm{m}$. (D) Statistical analysis of C. ${ }^{*} p<0.01$ versus vehicle group. ${ }^{* *} p<0.05$ versus vehicle group. (E) Determination of hemoglobin concentration by Drabkin's Reagent. ${ }^{*} p<0.01$ versus vehicle group. To see this illustration in color, the reader is referred to the web version of this article at www .liebertpub.com/ars 

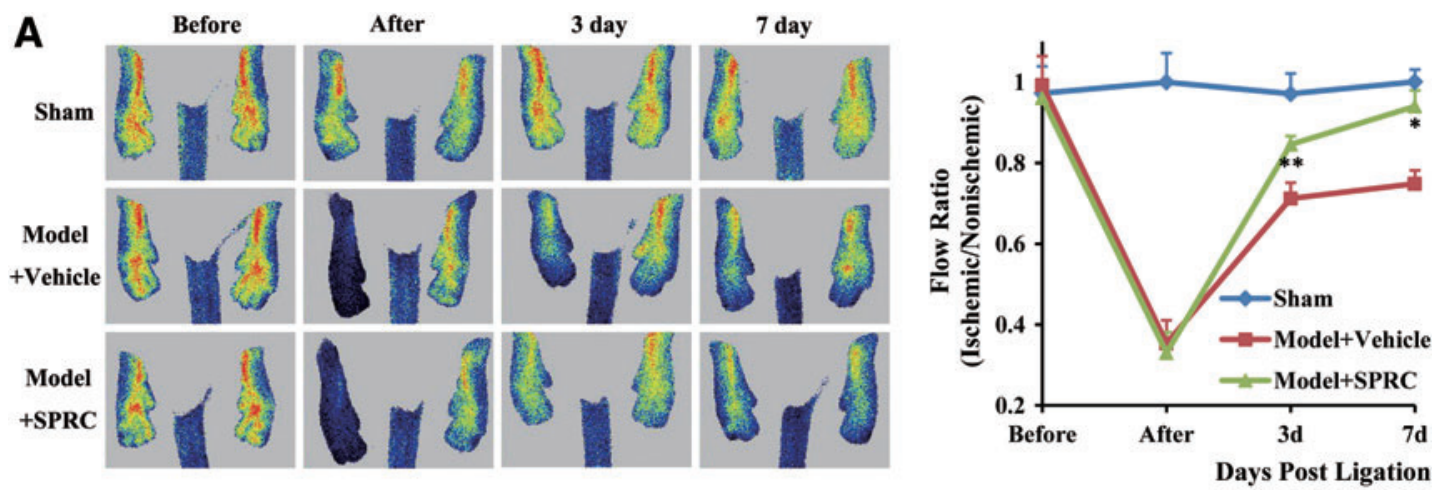

B
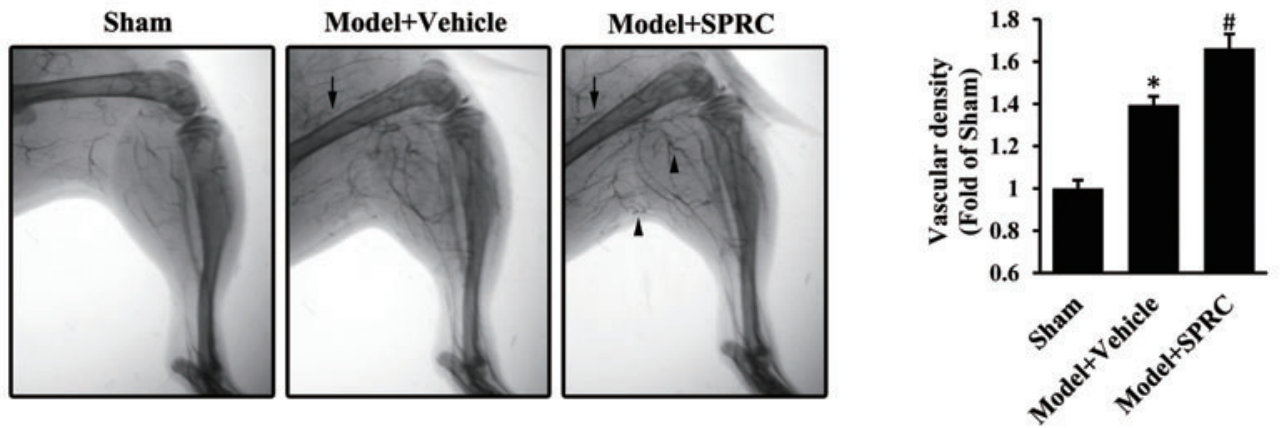

C

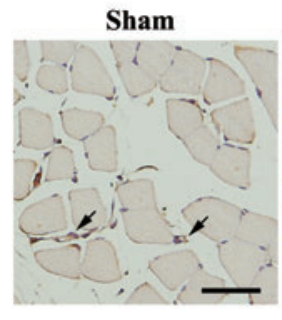

D

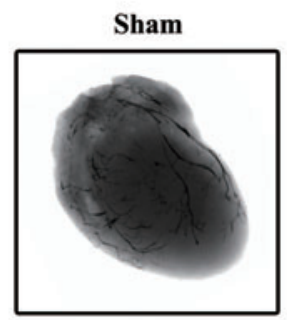

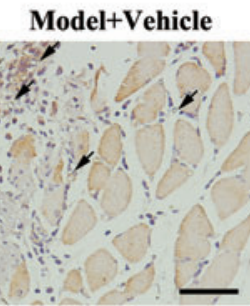
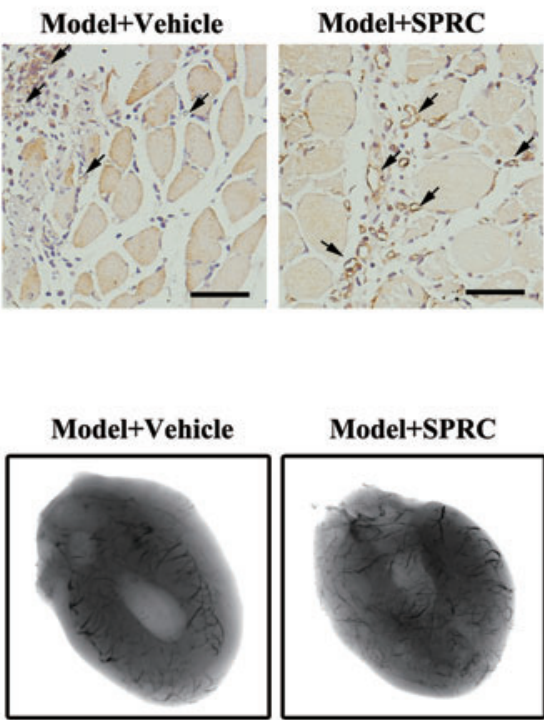

\section{Model+SPRC}

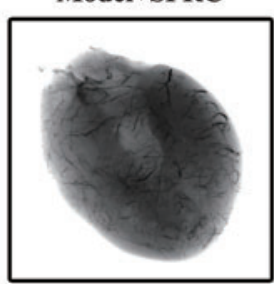

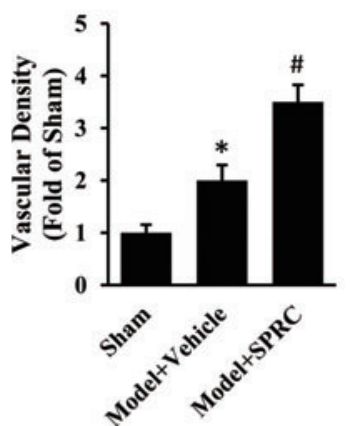

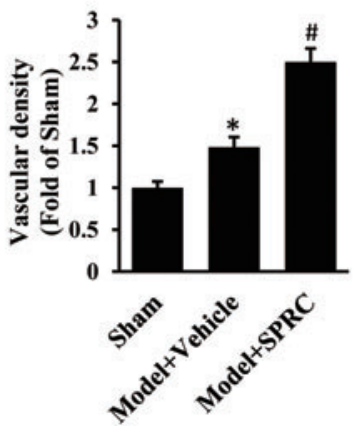

FIG. 4. SPRC promoted angiogenesis in vivo under ischemic condition. (A-C) After ligation of the left femoral artery, mice in the SPRC-treated group were injected intraperitoneally with SPRC ( $50 \mathrm{mg} / \mathrm{kg} /$ day), while mice in the vehicle model group received saline injections. (A) Mice were scanned by laser Doppler perfusion imaging before, immediately after, and 3 and 7 days after femoral artery ligation, and a flow ratio was determined by dividing the perfusion value of the ischemic hindlimb by that of the nonischemic one from the same animal. $n=6$ for each group. ${ }^{*} p<0.01$ versus vehicle model group, ${ }^{* *} p<0.05$ versus vehicle model group. (B) After treatment with SPRC or saline for 7 days, mice were anesthetized and injected with barium sulfate into the abdominal aorta to perform microangiography and to develop collateral vessels in the hindlimb. Solid arrow indicates ligation site, and the arrowhead indicates "corkscrew" collateral vessels. $n=6$ for each group. ${ }^{*} p<0.01$ versus sham group, ${ }^{*} p<0.01$ versus vehicle model group. (C) After treatment with SPRC or saline for 7 days, mice were anesthetized, and the gastrocnemius muscle was excised and stained with rabbit polyclonal CD31 antibody. Solid arrow indicates capillary vessels. Scale bar, $50 \mu \mathrm{m} . n=8$ for each group. ${ }^{*} p<0.01$ versus sham group, ${ }^{\#} p<0.01$ versus vehicle model group. (D) After ligation of the left coronary artery, rats in the SPRC-treated group were injected intraperitoneally with SPRC $(50 \mathrm{mg} / \mathrm{kg} /$ day) for 42 days, while rats in the vehicle model group received saline injections. Then, the rats were anesthetized and injected with barium sulfate into the left common carotid artery to perform microangiography. $n=5-7$ for each group. ${ }^{*} p<0.01$ versus sham group, ${ }^{\#} p<0.01$ versus vehicle model group. To see this illustration in color, the reader is referred to the web version of this article at www.liebertpub.com/ars 
A

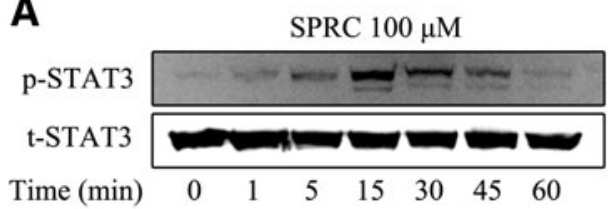

B

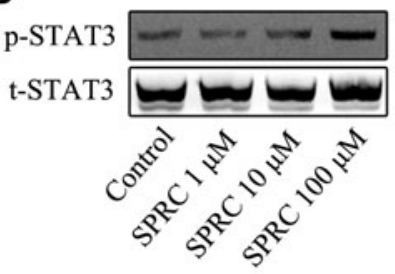

C $\begin{array}{ccccccl}0 & 0 & 0 & 0.1 & 0.1 & 0.1 & \operatorname{SPRC}(\mathrm{mM}) \\ 0 & 5 & 10 & 0 & 5 & 10 & \operatorname{WP} 1066(\mu \mathrm{M})\end{array}$

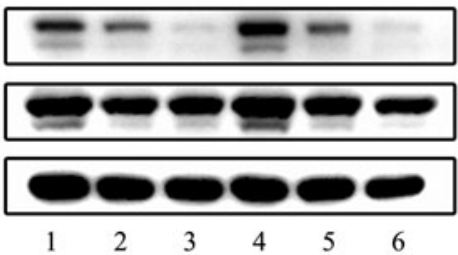

p-STAT3

t-STAT3

GAPDH
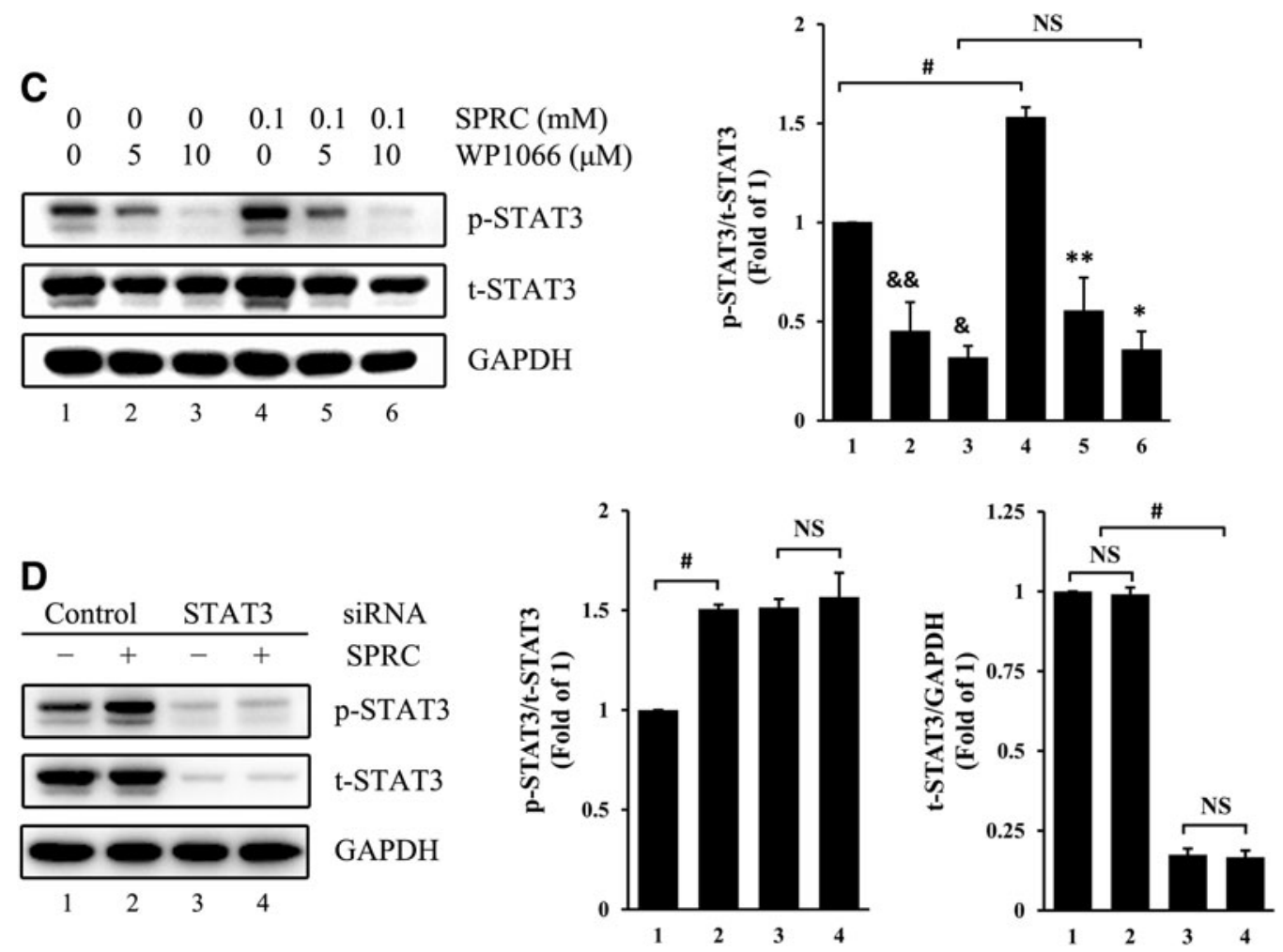

FIG. 5. SPRC-induced STAT3 phosphorylation. (A) Primary HUVEC were treated with SPRC (100 $\mu M)$ for a different time. (B) Primary HUVEC were treated with different SPRC concentrations for $15 \mathrm{~min}$. (C) Primary HUVEC were treated with WP1066 (5 or $10 \mu M)$, an inhibitor of STAT3, for $1.5 \mathrm{~h}$, followed by SPRC $(100 \mu M)$ for 20 min. * $p<0.01$ versus group 1 , ${ }^{\& \&} p<0.05$ versus group $1,{ }^{*} p<0.01$ versus group 4 , and ${ }^{* *} p<0.05$ versus group $4 .{ }^{\#} p<0.01$ between groups, as indicated. NS, nonsignificant $(p>0.05)$. (D) Primary HUVEC were treated with SPRC (100 $\mu M)$ for 20 min after STAT3 silencing, which was performed by siRNA transfection using an RNAiMAX kit for $72 \mathrm{~h}$. Control siRNA containing a scrambled sequence was used as a negative control. ${ }^{\#} p<0.01$ between groups, as indicated. NS, nonsignificant $(p>0.05)$. All experiments were repeated at least thrice.

stress, calcium overload, and inflammation (35). Therefore, a more microcirculation-friendly strategy should be explored to overcome and compensate for the shortcomings of conventional strategies. In previous research, fibroblast growth factor or VEGF was injected into the ischemic myocardium of patients with IHD as protein or plasmid, and this treatment promoted angiogenesis, reduced symptoms, and improved myocardial perfusion $(17,26)$. These results of angiogenesis-mediated therapy shed new light on IHD treatment (29).

In the past two decades, hydrogen sulfide has been extensively investigated for its biomedical effects. It is naturally produced in the human body and regulates metabolism and signal transduction to maintain homeostasis. In 2007, the proangiogenic effects of hydrogen sulfide were first reported by Zhu's group (4); then, in 2009, they were confirmed by Szabo's group (24). In 2010, Zhu's group published exciting results demonstrating that hydrogen sulfide promoted angiogenesis in a rat model of hindlimb ischemia, suggesting the potential utility of hydrogen sulfide-mediated proangiogenic therapy in ischemic disease (30). Although hydrogen sulfide has proven therapeutic potential, it diffuses quite readily and its targeted administration is, consequently, very difficult to achieve. Therefore, targeting a critical metabolic enzyme to modulate the endogenous hydrogen sulfide level may be a safe and effective direction for hydrogen sulfide-mediated drug therapy in the future (23). SPRC is a drug that was designed and developed by our group to target CTH for modulating endogenous hydrogen sulfide (Supplementary Fig. S8).

In this study, we demonstrated that SPRC promoted primary HUVEC proliferation, adhesion, migration, and tube formation as well as in vivo angiogenesis under both normal and ischemic conditions. In particular, using models of hindlimb and myocardium ischemia, we demonstrated the angiogenesis-mediated therapeutic potential of SPRC in IHD. 
A
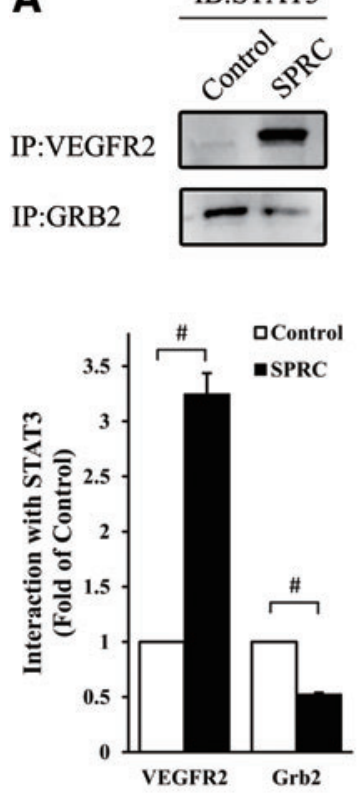

$\begin{array}{ccccccc}\text { C } & 0 & 0 & 0 & 0.1 & 0.1 & 0.1 \\ 0 & 5 & 10 & 0 & 5 & 10\end{array}$

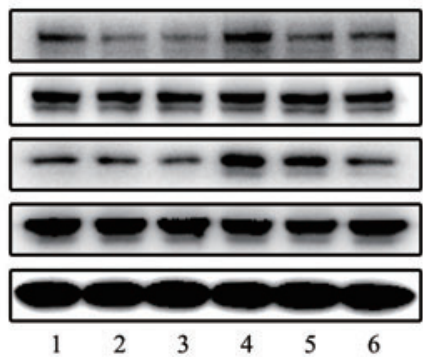

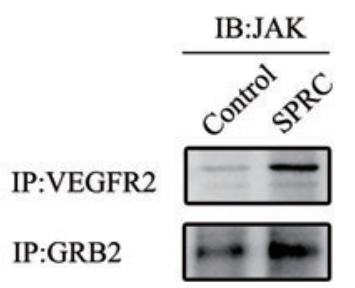

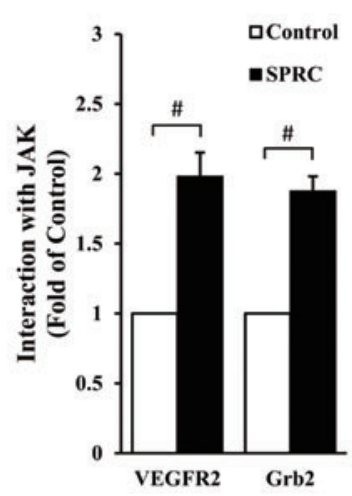

B
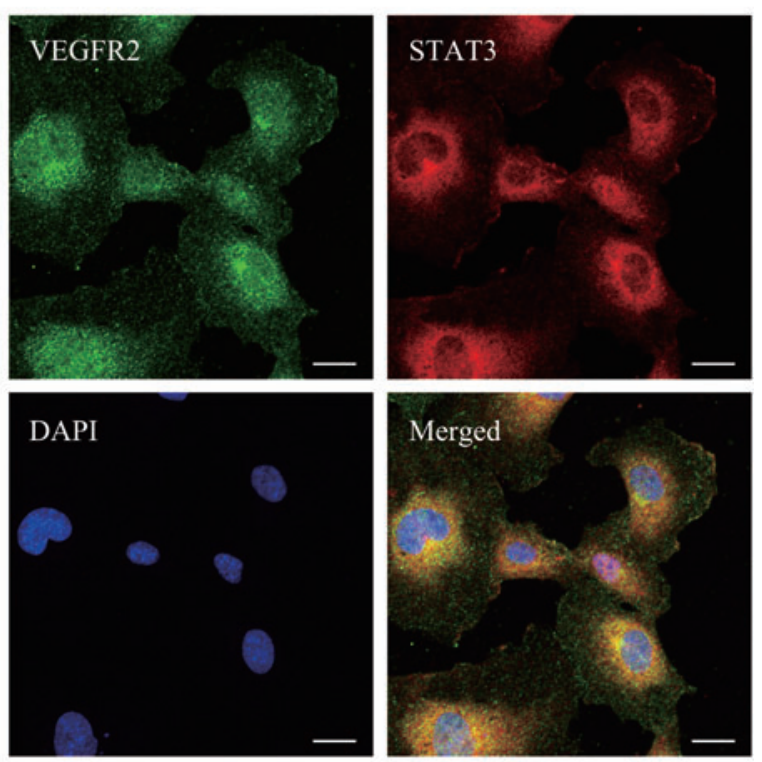
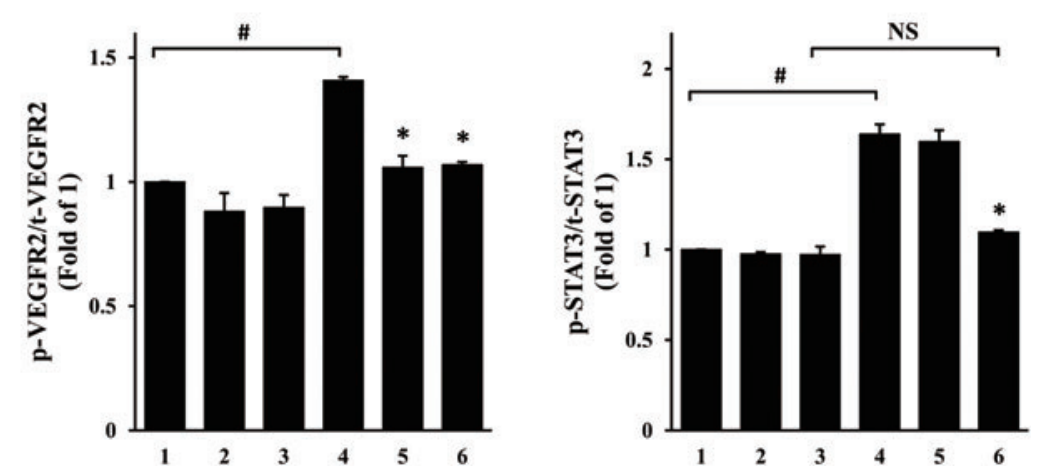

FIG. 6. The interaction between VEGFR2 and STAT3 was enhanced after SPRC treatment. (A) STAT3 or JAK protein levels in complexes immunoprecipitated with VEGFR2 or Grb2 antibody. HUV-EC-C were treated with SPRC (100 $\mu M)$ for $20 \mathrm{~min} .{ }^{\#} p<0.01$ between groups, as indicated. (B) Primary HUVEC were fixed and specifically stained with VEGFR2 antibody (green) and STAT3 antibody (red). DAPI (blue) indicates the nucleus. Scale bar, $20 \mu \mathrm{m}$. (C) Primary HUVEC were treated with SU5416 (5 or $10 \mu M)$, an inhibitor of VEGFR2, for $1 \mathrm{~h}$, followed by SPRC $(100 \mu M)$ for 20 min. ${ }^{*} p<0.01$ versus group 4. ${ }^{*} p<0.01$ between groups, as indicated. NS, nonsignificant $(p>0.05)$. All experiments were repeated at least thrice. To see this illustration in color, the reader is referred to the web version of this article at www.liebertpub.com/ars

In addition, in our recently published work, it was shown that SPRC could improve cardiac function as well as reduce infarct size and myocardial fibrosis in an IHD rat model (14). Compared with exogenous hydrogen sulfide-mediated proangiogenic therapy, the advantage of SPRC therapy is obvious. First, the safety problem of hydrogen sulfide is a concern. Hydrogen sulfide was first studied as a poisonous gas, and even in the human body, it is maintained at low levels. For these reasons, the exact dosage of exogenous hydrogen sulfide is difficult to determine. Second, hydrogen sulfide administration is problematic. In our experience, the exogenous hydrogen sulfide donor, NaHS, is necessary for frequent administration, for example every $4-6 \mathrm{~h}$, to maintain hydrogen sulfide at a certain level. In contrast, SPRC can be administered once daily.

STAT3 plays an important role in cell survival, and it is required for myocardial capillary growth after ischemic injury (12). STAT3 deletion in cardiomyocytes results in reduced capillarization of the left ventricle (12). In contrast, cardiacspecific STAT3 activation promotes vascular formation in the heart (22). Considering the vital role of STAT3 in angiogenesis, we first tested whether there was any effect of SPRC on STAT3 activity. We found that SPRC induced STAT3 phosphorylation in a time- and dose-dependent manner, and knockdown of STAT3 attenuated SPRC-induced angiogenesis in vitro. Taken together, these results suggested that STAT3 may be a key signaling molecule in the proangiogenic effects of SPRC. Therefore, we performed the present study to investigate how STAT3 was activated and how it activated downstream molecules.

First, we performed co-crystallization between SPRC and STAT3 to determine the presence of a direct interaction. SPRC is a small molecule and cysteine analog, whereas STAT3 is a protein with $\sim 769$ amino-acid residues. It was previously reported that cysteines introduced at residues $661 \mathrm{~A}$ and $663 \mathrm{~N}$ in a loop of the SH2 domain in STAT3 created a constitutively 
A

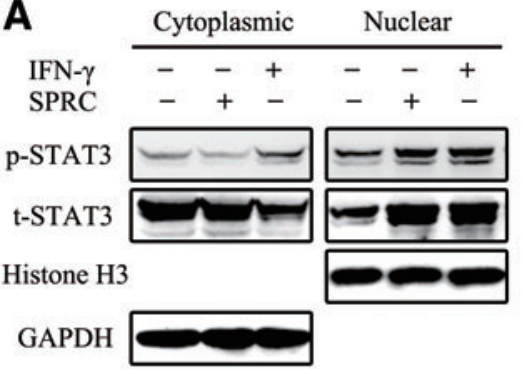

C

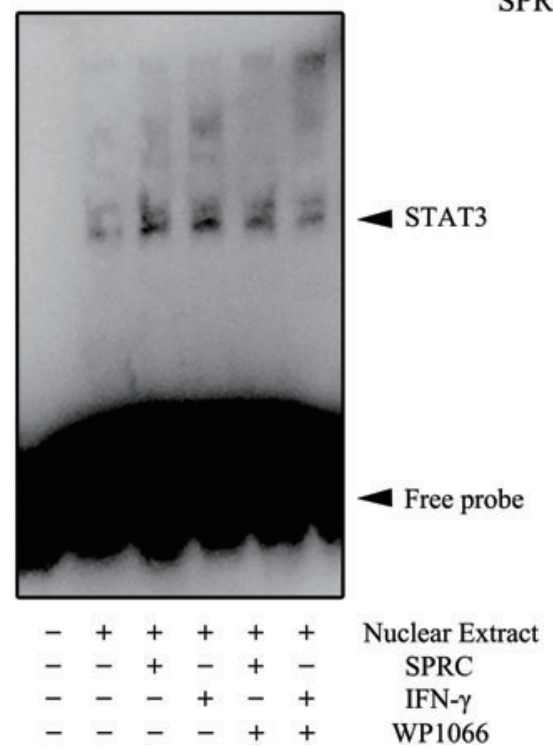

B

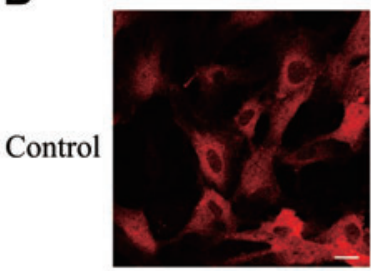

STAT3
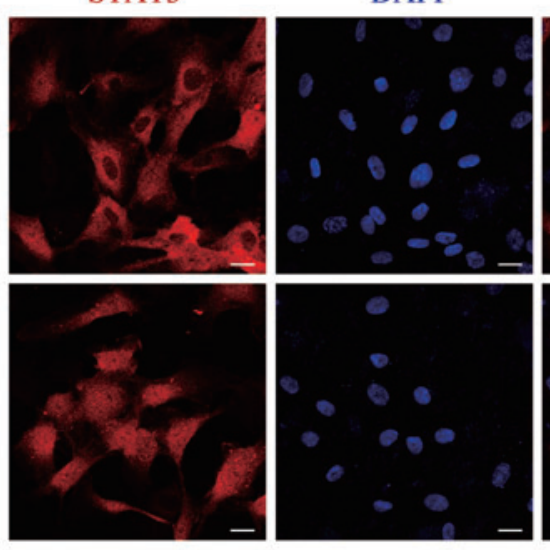

Merged
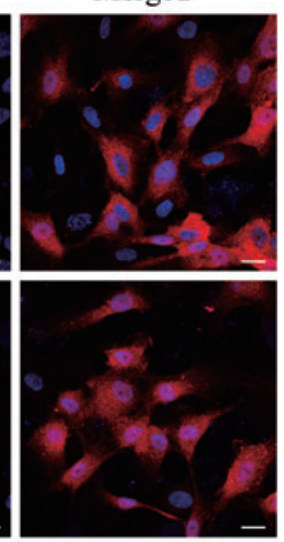

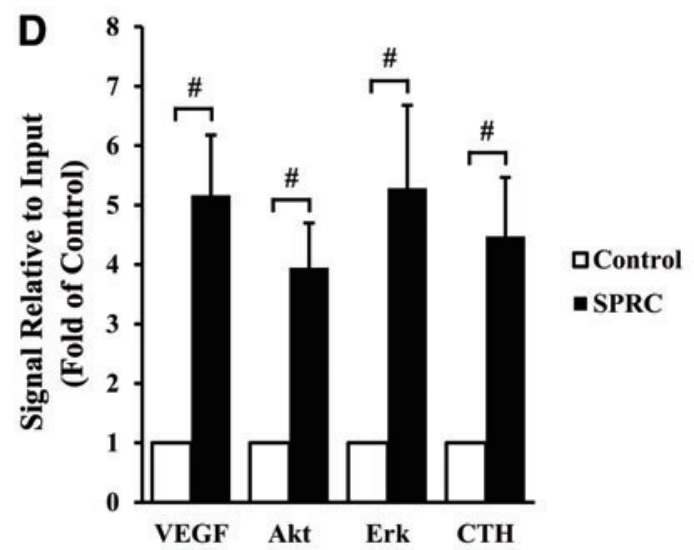

FIG. 7. SPRC induced the nuclear translocation of STAT3, followed by transcriptional activation. (A) HUV-EC-C were treated with SPRC $(100 \mu \mathrm{M})$ or interferon (IFN)- $\gamma(100 \mathrm{ng} / \mathrm{ml})$ for $45 \mathrm{~min}$, and then, cytoplasmic and nuclear proteins were isolated for Western blotting. GAPDH and histone H3 were used as a loading control for cytoplasmic and nuclear proteins, respectively. (B) Primary HUVEC were treated with SPRC $(100 \mu \mathrm{M})$ for $45 \mathrm{~min}$ and then stained with specific antibody (red) or DAPI (blue). Scale bar, $20 \mu \mathrm{m}$. (C) HUV-EC-C were treated with SPRC (100 $\mu \mathrm{M})$, IFN- $\gamma(100 \mathrm{ng} / \mathrm{ml})$, or WP1066 (10 $\mu$ M) for $45 \mathrm{~min}$. The nuclear extract was isolated and used for electrophoretic mobility shift assay. The shift band of STAT3 is indicated with a solid arrowhead. IFN- $\gamma$ was used as a positive control, and WP1066 was used to inhibit STAT3. (D) HUV-EC$\mathrm{C}$ were treated with SPRC $(100 \mu \mathrm{M})$ for $45 \mathrm{~min}$. The nuclear extract was isolated and used to perform chromatin immunoprecipitation with an STAT3 antibody. The change of downstream promoters was analyzed by real-time polymerase chain reaction. ${ }^{*} p<0.01$ between groups, as indicated. All experiments were repeated at least thrice. To see this illustration in color, the reader is referred to the web version of this article at www.liebertpub.com/ars

active protein (STAT3C) (3). In addition, the sulfhydryl in the cysteine is chemically active and susceptible to oxidization, serving an important structural role in many proteins (13). Therefore, we focused on STAT3 cysteine residues and analyzed the possible changes of surrounding electron densities after SPRC treatment. However, as shown in Supplementary Figure S3, after soaking in SPRC solution, the electron densities around the cysteine residue were still clear, and no significant extra-electron densities were observed.

After excluding a direct effect of SPRC on STAT3, we speculated that SPRC activated STAT3 via an endogenous hydrogen sulfide-mediated mechanism. In 2009, it was first reported that endogenous hydrogen sulfide could physiologically modify cysteines in many proteins by Ssulfhydration (18). Recently, our collaborating laboratory published that a newly discovered Cys1045-Cys1024 disulfide bond in VEGFR2 served as an intrinsic inhibitory motif and functioned as a molecular switch which could be broken by hydrogen sulfide to recover the active VEGFR2 conformation (28). As an RTK, VEGFR2 has been shown to directly activate STAT1 via protein interaction (2). These works inspired us to investigate whether VEGFR2/STAT3 interaction was involved in SPRC $\left(\mathrm{H}_{2} \mathrm{~S}\right)$ induced STAT3 activation. Co-immunoprecipitation revealed that the interaction between VEGFR2 and STAT3 was enhanced after SPRC treatment. Moreover, STAT3 was associated with VEGFR2, accompanied by dissociation from Grb2. Treatment with a VEGFR2 inhibitor demonstrated possible signal transduction from VEGFR2 to STAT3. The significance of these results is that it is a novel signal pathway of STAT3 activation, the $\mathrm{H}_{2} \mathrm{~S} /$ VEGFR2-mediated pathway (Fig. 8). Since the molecular switch for hydrogen sulfide is located in the intracellular domain of VEGFR2 and CTH is also localized to subcellular areas that are adjacent to the cell membrane (28), endogenous hydrogen sulfide generated by SPRC could be very close to the target motif of VEGFR2. Furthermore, STAT3 


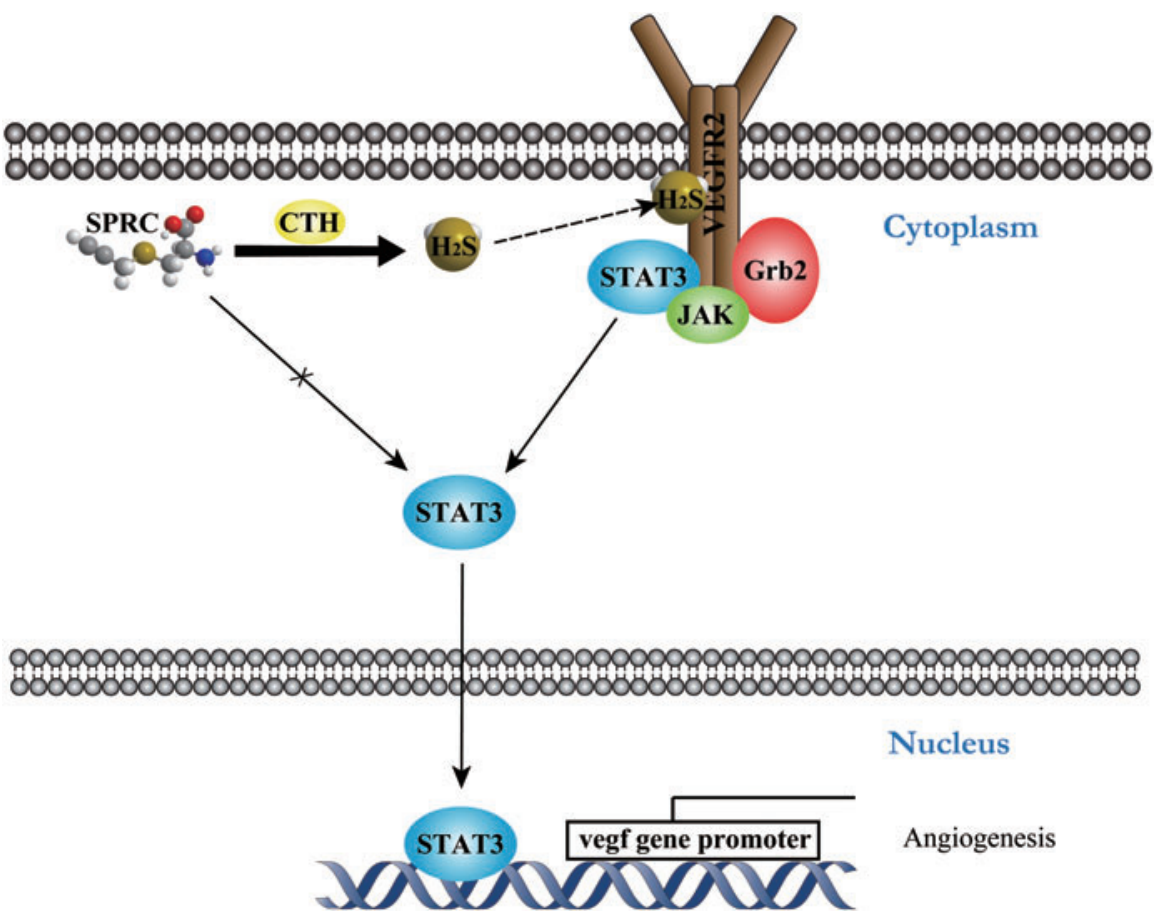

FIG. 8. Schematic illustration of $\mathrm{H}_{2} \mathrm{~S} /$ VEGFR2/STAT3-mediated mechanism in SPRC-induced angiogenesis. SPRC activates CTH to produce endogenous hydrogen sulfide. As a small gaseous molecule, hydrogen sulfide may diffuse to active VEGFR2 on the cell membrane. The signal transduction from VEGFR2 to STAT3 causes nuclear translocation of STAT3, which can lead to activation of the downstream promoters. A solid line indicates the clarified mechanism. A solid line with a cross indicates the excluded mechanism. A dashed line indicates the hypothetical mechanism. To see this illustration in color, the reader is referred to the web version of this article at www.liebertpub.com/ars co-localized with VEGFR2 in plasma, which may be the reason for the quick action of SPRC in the adhesion assay.

It was previously demonstrated that the nuclear translocation of STAT3 was essential for VEGF-induced endothelial cell migration and tube formation (34). In this work, we also observed a significant translocation of STAT3 from the cytosol to the nucleus after SPRC treatment. Furthermore, it was shown that STAT3 could bind to the Vegf promoter, which is directly regulated by STAT3 (19). Using ChIP analysis, we discovered that after SPRC treatment, the Vegf promoter and promoters of Akt, Erk, and Cth were activated by STAT3. Vegf promoter activation may be directly responsible for SPRCinduced angiogenesis, and up-regulated CTH transcription may provide a positive feedback signal in endothelial cells. In addition, it was previously reported that STAT3 and hypoxia inducible factor- $1 \alpha$ (HIF- $1 \alpha)$, which plays a critical role in regulating VEGF transcription, could simultaneously bind to the Vegf promoter, forming a molecular complex and activating transcription (11). Targeting STAT3 with a smallmolecule inhibitor could block HIF- $1 \alpha$ and VEGF expression in vitro and inhibit angiogenesis in vivo (33). Therefore, in future work, we will continue to investigate the synergistic effect of STAT3 and HIF- $1 \alpha$ on SPRC-induced transcriptional activation of the $V e g f$ promoter.

In conclusion, this is the first study suggesting that SPRC, a novel water-soluble modulator of endogenous hydrogen sulfide, can promote angiogenesis under both normal and ischemic conditions. Furthermore, we clarified an STAT3-mediated mechanism in SPRC-induced angiogenesis, including its interaction with VEGFR2 and transcriptional activation.

\section{Materials and Methods}

\section{Drugs and reagents}

SPRC was synthesized by the reaction of L-cysteine with propargyl bromide and then purified by recrystal- lization from an ethanol-water mixture (99\%) (32). PAG was purchased from Sigma (St. Louis, MO). VEGF and IFN- $\gamma$ were purchased from PeproTech (Rocky Hill, NJ). SU5416 and WP1066 were purchased from Calbiochem (Billerica, MA).

\section{Animals}

We obtained 6-week-old male C57BL/6 mice and 6- to 7week-old male Sprague-Dawley (SD) rats from the Department of Experimental Animals, Chinese Academy of Sciences (Shanghai, China). All animals received humane care, and all experimental procedures related to the animals complied with the "Guide for the Care and Use of Laboratory Animals" published by the National Institutes of Health (NIH) of the United States and were approved by the ethics committee of Experimental Research, Shanghai Medical College, Fudan University.

\section{Cell culture}

Primary HUVEC were purchased from ScienCell (Carlsbad, CA) and cultured in MCDB131 (PAA, Pasching, Austria) with 5\% FBS (Hyclone, Logan, UT), 1\% endothelial cell growth supplement (ScienCell), and 1\% penicillin streptomycin (Gibco, Grand Island, NY). Only cells from passages 4 to 6 were used for experiments. HUV-EC-C were obtained from ATCC (Rockville, MD) and cultured in DMEM (Gibco) with $10 \%$ FBS and $1 \%$ penicillin streptomycin.

\section{Cell viability assay}

Primary HUVEC were plated into 96-well plates and treated with different drug concentrations for $24 \mathrm{~h}$. Then, MTT (Amresco, Solon, $\mathrm{OH}$ ) was added, and cells were incubated for $4 \mathrm{~h}$. The absorbance at $570 \mathrm{~nm}$ in DMSO was read to assess cell viability. 


\section{Cell proliferation assay}

Primary HUVEC were plated into 96-well plates and treated with drugs for $24 \mathrm{~h}$. An EdU Assay Kit (RiboBio, Guangzhou, China) was used to dye the nuclei of proliferated cells red, which were then detected by fluorescence microscopy. The nuclei of all cells were also dyed blue with DAPI (4',6-diamidino-2-phenylindole).

\section{Cell adhesion assay}

Primary HUVEC were plated into 48-well plates that were precoated with $50 \mu \mathrm{g} / \mathrm{ml}$ of rat tail-derived collagen I (BD Biosciences, Franklin Lakes, NJ) and treated with different drug concentrations for $10 \mathrm{~min}$. Then, cells were washed with phosphate-buffered saline and fixed with $4 \%$ formaldehyde. Crystal violet staining solution (Beyotime, Shanghai, China) was used to stain the cells (4), and cells in three random fields from each well were counted to determine the average number.

\section{Wound-healing assay}

Primary HUVEC were plated into 48-well plates and treated with hydroxyurea (Sigma) to inhibit cell proliferation. Once a confluent cell monolayer formed, it was scraped with a yellow pipette tip to generate a scratch wound (4). Cells were treated with different drug concentrations for $24 \mathrm{~h}$, and photographed 0 and $24 \mathrm{~h}$ later with a Zeiss digital camera (Zeiss, Oberkochen, Germany). Wound areas were calculated to determine the migrated distance.

\section{Transwell migration assay}

Primary HUVEC were seeded into the upper compartment, which was precoated with $50 \mu \mathrm{g} / \mathrm{ml}$ collagen I, while the drug was added into the lower compartment. Cells were allowed to migrate for $8 \mathrm{~h}$ before they were fixed with $4 \%$ formaldehyde. The nonmigratory cells in the upper compartment were removed with a cotton swab, and the migrated cells were stained with crystal violet (4). Cells in three random fields from each well were counted to determine the average number.

\section{Tube formation assay}

Primary HUVEC were plated into 48-well plates that were precoated with Matrigel (Growth Factor Reduced, BD Biosciences) (4) and then treated with drugs. Tubular structures were photographed $6 \mathrm{~h}$ later and quantified by measuring the length of each tube using the software Image-Pro Plus 6 (Media Cybernetics, Rockville, MD). The tube length in three random fields from each well was calculated to determine the average length.

\section{Matrigel plug assay}

C57BL/ 6 mice were anesthetized by intraperitoneal $7 \%$ chloral hydrate, and they were injected subcutaneously with $500 \mu \mathrm{l}$ Matrigel (4). Matrigel premixed with VEGF (100 ng/ml) was used as a positive control. Drug-treated mice were injected intraperitoneally ( $50 \mathrm{mg} / \mathrm{kg} /$ day) daily for 7 days. Then, the mice were sacrificed, and the Matrigel plugs were removed. A few plugs were fixed overnight before embedding them in paraffin; the remaining plugs were immediately homogenized to determine hemoglobin concentration. Paraffin sections were soaked in hematoxylin-eosin solution (Beyotime) to stain cells that migrated into the Matrigel. Three random fields were captured by a Zeiss digital camera for each section. Cell number was analyzed by the software Image-Pro Plus 6. For immunohistochemistry, endothelial cells in the Matrigel were specifically stained by CD31 antibody according to the method described next. The hemoglobin concentrations were determined by Drabkin's Reagent (Sigma), and the values were normalized by the weight of the plugs (4).

\section{Rat aortic ring assay}

SD rats were anesthetized by intraperitoneal $7 \%$ chloral hydrate and dissected to obtain isolated aortae. The aorta was cut into segments, embedded in a Matrigel-containing environment, and cultured in MCDB131 with FBS and endothelial cell growth supplement (27). The aortic rings were treated with drugs for 6 days, and the medium and the drug were changed every 2 days. The aortic rings were photographed every other day. The areas of microvessels were calculated and statistically analyzed.

\section{Hindlimb ischemia model}

C57BL/ 6 mice were anesthetized by intraperitoneal 7\% chloral hydrate, and they were placed on a heating pad $\left(37^{\circ} \mathrm{C}\right)$ under an Olympus stereotactic microscope (Olympus, Tokyo, Japan). A longitudinal 5-mm incision was made, beginning at the inguinal crease along the left femoral vessels that were visible. The femoral artery was separated without damaging the vein or nerve and ligated with a 7.0-silk thread using triple surgical knots. Successful ligation was verified by laser Doppler perfusion imaging, which recorded colorcoded perfusion images showing perfusion deficit of the left paw after hindlimb ischemia (16). Sham operations were performed on animals by separating the femoral artery only, with no ligation. SPRC-treated mice were injected intraperitoneally $(50 \mathrm{mg} / \mathrm{kg} /$ day) daily for 7 days. The mice in the vehicle model group were injected intraperitoneally with saline.

\section{Myocardial ischemia model}

After being anesthetized by intraperitoneal 7\% chloral hydrate, SD rats were connected to an electrocardiograph recorder and treated by tracheal intubation. The left coronary artery was ligated permanently to induce myocardial ischemia as previously described (32). In brief, after cutting off the third rib, the left descending anterior coronary was ligated 2-3 mm near its origin with a 6.0-silk thread. Successful ligation was verified by the immediate color change in the ischemic area (anterior ventricular wall and the apex) and the occurrence of arrhythmias (ST-segment elevation). The chest was closed in layers, and the skin was sterilized with povidone iodine. At $24 \mathrm{~h}$ after surgery, the survival rate was $60 \%$. Thoracotomy was performed on sham-operated animals, and the pericardium only was opened, with no ligation around the left anterior descending artery. SPRC-treated rats were injected intraperitoneally ( $50 \mathrm{mg} / \mathrm{kg} /$ day) daily for 42 days. Rats in the vehicle model group were injected intraperitoneally with saline. 


\section{Laser doppler perfusion imaging}

Hindlimb perfusion was assessed using PeriCam PSI System-Blood Perfusion Imager (Perimed, Stockholm, Sweden) to scan mice that were placed on a heating plate and maintained at $37^{\circ} \mathrm{C}$. Perfusion was evaluated at the designated times (before, immediately after, and 3 and 7 days after femoral artery ligation), and a flow ratio was determined by dividing the perfusion value of ischemic hindlimb by that of the nonischemic one from the same animal (9).

\section{Immunohistochemistry}

On day 7 after femoral artery ligation, the mice were sacrificed, and the gastrocnemius muscle was excised, fixed, and embedded in paraffin. The endothelial cells in paraffin sections were recognized with a rabbit polyclonal CD31 antibody (Abcam, Cambridge, United Kingdom) followed by staining with a horseradish peroxidase (HRP)-conjugated secondary antibody, and diaminobenzidine substrate (Maixin, Fuzhou, China). Five random fields from each section were imaged with a Zeiss digital camera. Vascular density was analyzed with Image-Pro Plus 6 software.

\section{Microangiography}

For the hindlimb ischemia model, mice were anesthetized by $7 \%$ chloral hydrate on day 7 after femoral artery ligation, and catheters (PE-10; BD Biosciences) were implanted into the abdominal aorta at $0.5 \mathrm{~cm}$ to reach the iliac bifurcation. For the myocardial ischemia model, rats were anesthetized by $7 \%$ chloral hydrate on day 42 after left coronary artery ligation, and catheters (PE-50; BD Biosciences) were implanted into the left common carotid artery at $2.5-3 \mathrm{~cm}$ to reach the mitral valve. Then, heparinized saline $(10 \mathrm{U} / \mathrm{ml})$, nitroglycerine $(100 \mu \mathrm{g} / \mathrm{ml})$, and barium sulfate (size, $1 \mu \mathrm{m} ; 0.1 \mathrm{~g} / \mathrm{ml}$ ) were manually injected successively to perfuse the vessels in the hindlimb or heart $(10,30)$. The mice or isolated hearts of rats were placed in an X-ray chamber, and angiogram images were captured with the In Vivo FX PRO system (Carestream, Rochester, NY). Vascular density was determined by pixel analysis using the software ImageJ (NIH, Bethesda, MD).

\section{RNA interference}

Primary HUVEC were seeded in six-well plates the day before transfection. RNAiMAX (Invitrogen, Carlsbad, CA) was used to transfect $50 \mathrm{nM}$ STAT3 siRNA (Santa Cruz Biotechnology, Santa Cruz, CA) into HUVEC in OPTI-MEM I Reduced Serum Medium (Gibco) for 48 or $72 \mathrm{~h}$. Control siRNA containing a scrambled sequence was used as a negative control. The medium could be changed $6-8 \mathrm{~h}$ after transfection.

\section{Protein extraction and nuclear isolation}

M-PER Mammalian Protein Extraction Reagent (Pierce, Rockford, IL) was used to extract the total protein of cells. NEPER Nuclear and Cytoplasmic Extraction Reagents (Pierce) were used to extract the nuclear and cytoplasmic protein according to the manufacturer's instructions.

\section{Western blot}

Protein extracts were boiled with $5 \times$ loading buffer (Fermentas, Vilnius, Lithuania) and then loaded onto sodium dodecyl sulfate-polyacrylamide gels. The separated proteins were transferred to polyvinylidene fluoride (PVDF) membranes (Millipore, Billerica, MA), then blocked with 5\% nonfat dry milk in TBS (Amresco) containing 0.1\% Tween-20. Primary and secondary antibodies (Cell Signaling, Danvers, MA) were used to successively incubate the PVDF membrane. The HRP-conjugated protein was detected by Immobilon ${ }^{\mathrm{TM}}$ Western Chemiluminescent HRP Substrate (Millipore). The primary antibodies against p-STAT3, STAT3, p-VEGFR2, VEGFR2, JAK, Grb2, Histone H3, and GAPDH were purchased from Cell Signaling.

\section{Co-immunoprecipitation}

Cells in each dish were lysed with $400 \mu \mathrm{l}$ IP Lysis/Wash Buffer (Pierce). The protein extract was incubated with IPgrade primary antibody (Cell Signaling) in Spin ColumnScrew Cap (Pierce) with gentle mixing overnight at $4^{\circ} \mathrm{C}$. The next day, $20 \mu \mathrm{l}$ Protein G Agarose (Beyotime) was added to immunoprecipitate the bait-prey protein mixture. After centrifugation, the entire complex was boiled with $5 \times$ loading buffer to elute all proteins.

\section{Immunofluorescence}

Primary HUVEC were seeded onto cover glasses that were placed in six-well plates the day before treatment. After fixation with $4 \%$ formaldehyde and blocking with 5\% BSA (Amresco), cells were incubated with primary antibody against STAT3 or VEGFR2 (Cell Signaling) overnight at $4^{\circ} \mathrm{C}$, followed by Alexa Fluor 594- or 488-conjugated secondary antibody (Molecular Probe, Grand Island, NY) for $2 \mathrm{~h}$ before they were imaged with confocal laser scanning microscopy (Zeiss).

\section{Electrophoretic mobility shift assay}

A LightShift ${ }^{\circledR}$ Chemiluminescent EMSA Kit (Pierce) was used to perform EMSA according to the manufacturer's instructions. The sequence of STAT3 DNA probe (Santa Cruz) was listed as follows:

5'-GAT CCT TCT GGG AAT TCC TAG ATC-3'

3'-CTA GGA AGA CCC TTA AGG ATC TAG-5'

\section{Chromatin immunoprecipitation}

ChIP was performed with an Agarose ChIP Kit (Pierce) according to the manufacturer's instructions. A ChIP-grade primary antibody against STAT3 was purchased from Cell Signaling. Immunoprecipitated DNA was purified with Qiagen DNA Clean-Up Column and then quantitated by real-time polymerase chain reaction (PCR).

\section{Real-time PCR}

Purified DNA from ChIP was mixed with promoter primer and $2 \times$ Maxima SYBR Green qPCR Master Mix (Fermentas). PCR was performed by iCycler iQ5 (Bio-Rad, Hercules, CA) at an annealing/extension temperature of $62^{\circ} \mathrm{C}$. The promoter primers sequences were as follows:

\footnotetext{
Vegf: 5'-CTGGCCTGCAGACATCAAAGTGAG-3' 5'-CTTCCCGTTCTCAGCTCCACAAAC-3'

Cth: 5'-CCGCAGGGACTAACACCACTTG-3' 5'-CCCGCTCCTTATTGGCTGACAG-3'
} 


\section{Akt: 5'-GGATAAAGTGTGCTCAGGTGAGGG-3' 5'-ATTCTAGGCTTAGAGCCTCCAGCC-3' \\ Erk: 5'-GGCGTATCCTCTCAGTGTCTCC-3' 5'-GGCAGAAACCGAAAGGCATGAC-3'}

\section{Statistical analysis}

Quantitative data are presented as mean \pm SEM. Differences between groups were analyzed using Student's $t$-test when only two groups were compared or assessed by oneway analysis of variance with Tukey's post-hoc test when more than two groups were compared. A probability value of no more than 0.05 was considered statistically significant.

\section{Acknowledgments}

This work was supported by the National Basic Research Program of China (973 Program, No. 2010CB912603), the Key Program of Shanghai Committee of Science and Technology in China (No. 10431900100), the National Science and Technology Major Project (No. 2012Z X 09501001-001-003), and the Innovation Fund for Graduate Students of Fudan University (No. EZF301302). The authors thank Jian Zhu and his colleagues at Shanghai Medicilon Inc. for performing protein expression, purification studies, and crystallization analyses. They also thank Beibei Tao for the excellent technical assistance rendered.

\section{Author Disclosure Statement}

No competing financial interests exist.

\section{References}

1. Abe $\mathrm{K}$ and Kimura $\mathrm{H}$. The possible role of hydrogen sulfide as an endogenous neuromodulator. J Neurosci 16: 1066-1071, 1996.

2. Bartoli M, Gu XL, Tsai NT, Venema RC, Brooks SE, Marrero $\mathrm{MB}$, and Caldwell RB. Vascular endothelial growth factor activates STAT proteins in aortic endothelial cells. J Biol Chem 275: 33189-33192, 2000.

3. Bromberg JF, Wrzeszczynska MH, Devgan G, Zhao YX, Pestell RG, Albanese C, and Darnell JE. Stat3 as an oncogene. Cell 98: 295-303, 1999.

4. Cai WJ, Wang MJ, Moore PK, Jin HM, Yao T, and Zhu YC. The novel proangiogenic effect of hydrogen sulfide is dependent on Akt phosphorylation. Cardiovasc Res 76: 29-40, 2007.

5. Calvert JW, Jha S, Gundewar S, Elrod JW, Ramachandran A, Pattillo CB, Kevil CG, and Lefer DJ. Hydrogen Sulfide Mediates Cardioprotection Through Nrf2 Signaling. Circ Res 105: 365-U105, 2009.

6. Carmeliet P. Angiogenesis in life, disease and medicine. Nature 438: 932-936, 2005.

7. Chalothorn D, Clayton JA, Zhang H, Pomp D, and Faber JE. Collateral density, remodeling, and VEGF-A expression differ widely between mouse strains. Physiol Genomics 30: 179-191, 2007.

8. Ferrara N, Gerber HP, and LeCouter J. The biology of VEGF and its receptors. Nat Med 9: 669-676, 2003.

9. Frontini MJ, Nong Z, Gros R, Drangova M, O'Neil C, Rahman MN, Akawi O, Yin H, Ellis CG, and Pickering JG. Fibroblast growth factor 9 delivery during angiogenesis produces durable, vasoresponsive microvessels wrapped by smooth muscle cells. Nat Biotechnol 29: 421-427, 2011.

10. Givvimani S, Tyagi N, Sen U, Mishra PK, Qipshidze N, Munjal C, Vacek JC, Abe OA, and Tyagi SC. MMP-2/TIMP-
2/TIMP-4 versus MMP-9/TIMP-3 in transition from compensatory hypertrophy and angiogenesis to decompensatory heart failure. Arch Physiol Biochem 116: 63-72, 2010.

11. Gray MJ, Zhang J, Ellis LM, Semenza GL, Evans DB, Watowich SS, and Gallick GE. HIF- $1 \alpha$, STAT3, CBP/p300 and Ref-1/APE are components of a transcriptional complex that regulates Src-dependent hypoxia-induced expression of VEGF in pancreatic and prostate carcinomas. Oncogene 24: 3110-3120, 2005.

12. Hilfiker-Kleiner D, Hilfiker A, Fuchs M, Kaminski $K$, Schaefer A, Schieffer B, Hillmer A, Schmiedl A, Ding ZP, Podewski E, Poli V, Schneider MD, Schulz R, Park JK, Wollert KC, and Drexler H. Signal transducer and activator of transcription 3 is required for myocardial capillary growth, control of interstitial matrix deposition, and heart protection from ischemic injury. Circ Res 95: 187-195, 2004.

13. Holmgren A. Thioredoxin structure and mechanism: conformational changes on oxidation of the active-site sulfhydryls to a disulfide. Structure 3: 239-243, 1995.

14. Huang C, Kan J, Liu X, Ma F, Tran BH, Zou Y, Wang S, and $\mathrm{Zhu}$ YZ. Cardioprotective effects of a novel hydrogen sulfide agent-controlled release formulation of s-propargyl-cysteine on heart failure rats and molecular mechanisms. PLoS One 8: e69205, 2013.

15. Levy DE and Darnell JE. STATs: Transcriptional control and biological impact. Nat Rev Mol Cell Biol 3: 651-662, 2002.

16. Limbourg A, Korff T, Napp LC, Schaper W, Drexler H, and Limbourg FP. Evaluation of postnatal arteriogenesis and angiogenesis in a mouse model of hind-limb ischemia. Nat Protoc 4: 1737-1748, 2009.

17. Losordo DW, Vale PR, Symes JF, Dunnington CH, Esakof DD, Maysky M, Ashare AB, Lathi K, and Isner JM. Gene therapy for myocardial angiogenesis - Initial clinical results with direct myocardial injection of phVEGF(165) as sole therapy for myocardial ischemia. Circulation 98: 2800-2804, 1998.

18. Mustafa AK, Gadalla MM, Sen N, Kim S, Mu WT, Gazi SK, Barrow RK, Yang GD, Wang R, and Snyder SH. H2S Signals Through Protein S-Sulfhydration. Sci Signal 2, 2009.

19. Niu GL, Wright KL, Huang M, Song LX, Haura E, Turkson J, Zhang SM, Wang TH, Sinibaldi D, Coppola D, Heller R, Ellis LM, Karras J, Bromberg J, Pardoll D, Jove R, and Yu H. Constitutive Stat3 activity up-regulates VEGF expression and tumor angiogenesis. Oncogene 21: 2000-2008, 2002.

20. Olsson AK, Dimberg A, Kreuger J, and Claesson-Welsh L. VEGF receptor signalling-in control of vascular function. Nat Rev Mol Cell Biol 7: 359-371, 2006.

21. Organization WH. The 10 Leading Causes of Death in the World, 2000 and 2011. Geneva: World Health Organization, 2013.

22. Osugi T, Oshima Y, Fujio $Y$, Funamoto M, Yamashita A, Negoro S, Kunisada K, Izumi M, Nakaoka Y, Hirota H, Okabe M, Yamauchi-Takihara K, Kawase I, and Kishimoto T. Cardiac-specific activation of signal transducer and activator of transcription 3 promotes vascular formation in the heart. J Biol Chem 277: 6676-6681, 2002.

23. Pan LL, Liu XH, Gong QH, Yang HB, and Zhu YZ. Role of cystathionine $\gamma$-lyase/hydrogen sulfide pathway in cardiovascular disease: a novel therapeutic strategy? Antioxid Redox Signal 17: 106-118, 2012.

24. Papapetropoulos A, Pyriochou A, Altaany Z, Yang GD, Marazioti A, Zhou ZM, Jeschke MG, Branski LK, Herndon DN, Wang R, and Szabo C. Hydrogen sulfide is an endogenous stimulator of angiogenesis. Proc Natl Acad Sci U S A 106: 21972-21977, 2009. 
25. Predmore BL, Lefer DJ, and Gojon G. Hydrogen sulfide in biochemistry and medicine. Antioxid Redox Signal 17: 119140, 2012.

26. Schumacher B, Pecher P, von Specht BU, and Stegmann T. Induction of neoangiogenesis in ischemic myocardium by human growth factors - First clinical results of a new treatment of coronary heart disease. Circulation 97: 645-650, 1998.

27. Srivastava K, Kundumani-Sridharan V, Zhang B, Bajpai AK, and Rao GN. 15 (S)-Hydroxyeicosatetraenoic Acid-Induced Angiogenesis Requires STAT3-Dependent Expression of VEGF. Cancer Res 67: 4328-4336, 2007.

28. Tao BB, Liu SY, Zhang CC, Fu W, Cai WJ, Wang Y, Shen Q, Wang MJ, Chen Y, Zhu YZ, and Zhu YC. VEGFR2 functions as an H2S-targeting receptor protein kinase with its novel Cys1045-Cys1024 disulfide bond serving as a specific molecular switch for hydrogen sulfide actions in vascular endothelial cells. Antioxid Redox Signal 19: 448-464, 2012.

29. van der Laan AM, Piek JJ, and van Royen N. Targeting angiogenesis to restore the microcirculation after reperfused MI. Nat Rev Cardiol 6: 515-523, 2009.

30. Wang MJ, Cai WJ, Li N, Ding YJ, Chen Y, and Zhu YC. The Hydrogen Sulfide Donor NaHS Promotes Angiogenesis in a Rat Model of Hind Limb Ischemia. Antioxid Redox Signal 12: 1065-1077, 2010.

31. Wang Q, Liu HR, Mu Q, Rose P, and Zhu YZ. S-propargylcysteine protects both adult rat hearts and neonatal cardiomyocytes from ischemia/hypoxia injury: the contribution of the hydrogen sulfide-mediated pathway. J Cardiovasc Pharmacol 54: 139-146, 2009.

32. Wang $Q$, Wang XL, Liu HR, Rose P, and Zhu YZ. Protective effects of cysteine analogues on acute myocardial ischemia: novel modulators of endogenous $\mathrm{H}(2) \mathrm{S}$ production. Antioxid Redox Signal 12: 1155-1165, 2010.

33. Xu Q, Briggs J, Park S, Niu GL, Kortylewski M, Zhang SM, Gritsko T, Turkson J, Kay H, Semenza GL, Cheng JQ, Jove R, and $\mathrm{Yu}$ H. Targeting Stat3 blocks both HIF-1 and VEGF expression induced by multiple oncogenic growth signaling pathways. Oncogene 24: 5552-5560, 2005.

34. Yahata Y, Shirakata Y, Tokumaru S, Yamasaki K, Sayama K, Hanakawa Y, Detmar M, and Hashimoto K. Nuclear translocation of phosphorylated STAT3 is essential for vascular endothelial growth factor-induced human dermal microvascular endothelial cell migration and tube formation. J Biol Chem 278: 40026-40031, 2003.

35. Yellon DM and Hausenloy DJ. Mechanisms of disease: myocardial reperfusion injury. N Engl J Med 357: 1121-1135, 2007.

36. Yla-Herttuala $S$ and Alitalo K. Gene transfer as a tool to induce therapeutic vascular growth. Nat Med 9: 694-701, 2003.

37. Zhao WM, Zhang J, Lu YJ, and Wang R. The vasorelaxant effect of $\mathrm{H} 2 \mathrm{~S}$ as a novel endogenous gaseous K-ATP channel opener. EMBO J 20: 6008-6016, 2001.
38. Zhong Z, Wen ZL, and Darnell JE. Stat3: a STAT family member activated by tyrosine phosphorylation in response to epidermal growth-factor and interleukin-6. Science 264: 95-98, 1994.

39. Zhu YZ, Wang ZJ, Ho PY, Loke YY, Zhu YC, Huang SH, Tan CS, Whiteman M, Lu J, and Moore PK. Hydrogen sulfide and its possible roles in myocardial ischemia in experimental rats. J Appl Physiol 102: 261-268, 2007.

Address correspondence to:

Prof. Yi Zhun Zhu

Department of Pharmacology

School of Pharmacy and Institutes of Biomedical Sciences

Fudan University

826 Zhangheng Road

201203 Shanghai

China

E-mail: zhuyz@fudan.edu.cn; phczhuyz@nus.edu.sg

Date of first submission to ARS Central, May 27, 2013; date of final revised submission, October 23, 2013; date of acceptance, November 1, 2013.

$\quad$ Abbreviations Used
ChIP $=$ chromatin immunoprecipitation
CTH, CSE $=$ cystathionine- $\gamma$-lyase
EMSA $=$ electrophoretic mobility shift assay
Grb2 $=$ growth factor receptor-bound protein 2
HIF- $\alpha=$ hypoxia inducible factor- $1 \alpha$
HUVEC $=$ human umbilical vein endothelial cells
HUV-EC-C $=$ transformed cells of human umbilical
vein endothelium
IFN $=$ interferon
IHD $=$ ischemic heart disease
JAK $=$ interleukin- 6
NF $=$ janus kinase
PAG $=$ propargylglycine
PCI $=$ percutaneous coronary intervention
PCR $=$ polymerase chain reaction
PVDF $=$ polyvinylidene fluoride
RTK $=$ receptor tyrosine kinase
SPRC $=$ S-propargyl-cysteine
STAT3 $=$ signal transducer and activator of
transcription 3
VEGF $=$ vascular endothelial growth factor
VEGFR $=$ vascular endothelial growth factor receptor 2

